\title{
Electrospun aniline-tetramer-co-polycaprolactone fibers for conductive, biodegradable scaffolds
}

\begin{abstract}
A. G. Guex, Department of Materials, Department of Bioengineering, Institute of Biomedical Engineering, Imperial College London, Prince Consort Road, London SW7 2AZ, UK; National Heart and Lung Institute, Imperial College London, Du Cane Road, London W12 ONN, UK; Empa, Swiss Federal Laboratories for Materials Science and Technology, Laboratory for Biointerfaces, and Laboratory for Biomimetic Membranes and Textiles, Lerchenfeldstrasse 5, 9014 St. Gallen, Switzerland

C. D. Spicer, A. Armgarth, and A. Gelmi, Department of Materials, Department of Bioengineering, Institute of Biomedical Engineering, Imperial College London, Prince Consort Road, London SW7 2AZ, UK

E. J. Humphrey, C. M. Terracciano, and S. E. Harding, National Heart and Lung Institute, Imperial College London, Du Cane Road, London W12 ONN, UK

M. M. Stevens, Department of Materials, Department of Bioengineering, Institute of Biomedical Engineering, Imperial College London, Prince Consort Road, London SW7 2AZ, UK
\end{abstract}

Address all correspondence to M. M. Stevens at m.stevens@imperial.ac.uk

(Received 21 April 2017; accepted 9 June 2017)

\section{Abstract}

Conjugated polymers have been proposed as promising materials for scaffolds in tissue engineering applications. However, the restricted processability and biodegradability of conjugated polymers limit their use for biomedical applications. Here we synthesized a block-co-polymer of aniline tetramer and PCL (AT-PCL), and processed it into fibrous non-woven scaffolds by electrospinning. We showed that fibronectin (Fn) adhesion was dependent on the AT-PCL oxidative state, with a reduced Fn unfolding length on doped membranes. Furthermore, we demonstrated the cytocompatibility and potential of these membranes to support the growth and osteogenic differentiation of MC3T3-E1 cells over 21 days.

\section{Introduction}

For several decades, cell therapies and tissue engineering have been increasingly considered as new therapeutic strategies to treat large bone defects or non-union fractions. ${ }^{[1-3]}$ Recreating the complexity of native tissue through three-dimensional (3D) constructs, cell-material biografts, or biomaterials in general remains incredibly challenging. ${ }^{[4]}$ Importantly, cell adhesion, proliferation, differentiation, and subsequent tissue formation, as well as clinical outcome, are strongly dependent on the choice of scaffold design, the ability of cells to interact with the material, and the successful creation of an artificial microenvironment. Ideally scaffolds should recreate the architectural, chemical, mechanical, and electrical properties of the host tissue. ${ }^{[5]}$ A variety of tissues, including bone, are susceptible to electrical stimulation, and the inherent piezoelectric properties of bone tissue has led to the hypothesis that electroactive materials could be suitable for promoting in vivo bone repair and osteogenesis. ${ }^{[6,7]}$ To this end, conjugated organic polymers such as polypyrrole (PPy), polyaniline (PANi), or poly(3,4-ethylenedioxythiophene) (PEDOT) have been increasingly considered for the development of novel electroactive scaffolds due to their reported cytocompatibility. ${ }^{[8-10]}$ After implantation of such materials in vivo, cell adhesion to a conjugated polymer membrane will be in part supported and maintained by the extracellular matrix (ECM) deposited by cells in the vicinity, and proteins present in the cell media serum. The initial adsorbance of proteins to a material is therefore critical in determining and controlling cell adhesion, immune response, and overall performance of an implant, and is therefore a key consideration during biomaterial design. ${ }^{[1]}$ This is particularly the case when using conjugated polymer-based materials, where the oxidation state of the polymer strongly determines material properties and is responsive to the local environment. Upon doping of a conjugated polymer, a change in electronic configuration or redox state not only leads to the conduction of electrical current, but furthermore alters intermolecular interactions with proteins and significantly influences protein adsorption. ${ }^{[12,13]}$ For example, fibronectin (Fn), a common protein of the ECM which promotes cellular adhesion, has been demonstrated to interact with cells in a protein conformation dependent manner. ${ }^{[14]}$ As such, the redox state-dependent conformation and adhesion of Fn to a conjugated substrate is key in determining interactions at the biological interface. ${ }^{[15]}$ Hence, by measuring the protein adsorption profile we can begin to elucidate the effect of conjugated polymers on the promotion of cellular adhesion. The ability to tune redox states by choice of dopant or synthesis strategy thereby renders conjugated polymers 
promising candidates for studying controlled protein adsorption and the resultant cell response.

Despite these promising properties of conjugated polymers, their application in tissue engineering remains hindered by many severe drawbacks associated with their use. For example, their low solubility in both aqueous and organic solvents, reduced processability, high heterogeneity when synthesized via traditional routes, lack of cell adhesion sites, and nonbiodegradability are severely limiting. As a result, recent research has focused on the design of biodegradable conductive scaffolds by restricting conjugation to oligomers of 4 to 8 repeating units. ${ }^{[16,17]}$ Oligomers provide a versatile route for customized, end-functionalized units with tuneable solubility and processability ${ }^{[18]}$ that allow for significantly enhanced freedom and flexibility with respect to scaffold design and integration into higher order architectures and biocomposites, while maintaining a significant degree of electroactivity. For example, a tetrameric oligomer of aniline exhibits redox and doping chemistries similar to poly(aniline) and has been shown to present even higher stability during charging-discharging cycling processes due to its shorter chain length and lack of long-chain swelling. ${ }^{[19]}$ Further, oligomers can be synthesized at defined molecular weights, leading to monodisperse products that will result in more concise and reproducible material design. ${ }^{[18]}$ Substantial efforts have gone into the development of aniline tetramer ${ }^{[20-22]}$ or aniline pentamer ${ }^{[7]}$ based scaffolds, with conjugated units being grafted to gelatine, poly(lactic acid) (PLA), poly(ethylene glycol), or poly(caprolactone) (PCL).

Preliminary investigations into the cytocompatibility, biodegradation or in vivo response of such materials have been reported, but open questions with respect to the application of such scaffolds in bone tissue engineering still remain. ${ }^{[23-25]}$ The aim of the current study therefore was to produce electrospun membranes based on aniline tetramer and evaluate these membranes in vitro. Our study in particular addressed the question of long-term studies and whether these biodegradable, electrically active membranes can successfully be used as substrates for bone tissue engineering. Our study further underpins the challenges encountered in the field and emphasizes on the need of in depth characterization and defined conditions to meet the demand for highly complex in vitro model systems.

\section{Material and methods}

Unless stated otherwise, all chemicals were purchased from Sigma-Aldrich, UK and used without further purification. Cell culture consumables and supplements were from Life Technologies, Thermo Fisher, UK, sterile, and used as provided.

\section{Aniline tetramer-co-poly(caprolactone) synthesis and characterization}

Aniline tetramer-co-poly(caprolactone) (AT-PCL) in the emeraldine base (EB) state was prepared in a two-step synthesis, as previously reported ${ }^{[22]}$ and detailed in supporting information (SI). AT-PCL was obtained at a molecular weight of $\sim 1300$ $\mathrm{g} / \mathrm{mol}$ [estimated based on nuclear magnetic resonance
(NMR) spectra as described in the SI]. The reaction scheme and NMR spectra are displayed in Supplementary Figs. S1 and S2. Optoelectronic activity was assessed by absorption spectroscopy. AT-PCL was dissolved in dimethyl sulfoxide (DMSO) and doped with phytic acid at increasing vol\% $(0.005-0.1 \mathrm{M})$. Spectra were recorded in the wavelength range of $200-1000 \mathrm{~nm}$ at a step size of $10 \mathrm{~nm}$ (Mithras2 Plate reader, Berthold Technologies, Germany).

\section{Electrospinning}

AT-PCL was blended with high molecular weight PCL $\left(M_{\mathrm{w}} \sim\right.$ $70,000-90,000 \mathrm{Da})$ at $\mathrm{w} / \mathrm{w}$ ratios of $25 \%, 50 \%$, or $75 \%$ ATPCL to PCL, and dissolved in a mixture of $\mathrm{CHCl}_{3}: \mathrm{CH}_{3} \mathrm{OH}$ $(9: 1 \mathrm{vol} \%)$. Solutions were doped with phytic acid to a final concentration of $0.02 \mathrm{M}$. Polymer solutions were processed into fibrous non-wovens on a custom built electrospinning device, as detailed in SI. ${ }^{[26]}$ All scaffolds were spun at 10/ $-5 \mathrm{kV}, 20 \mu \mathrm{L} / \mathrm{min}$ flow rate, and a needle to collector distance of $15 \mathrm{~cm}$. Fiber diameters were measured with ImageJ software, based on scanning electron micrographs recorded at three random spots on the membrane (nine images, 100 fibers per condition). Electrical characterization of electrospun membranes was carried out using a Keithley 2400 source meter, controlled by customized LabView software. The sheet resistance of dry, fibrous mats, doped in $0.1 \mathrm{M}$ hydrochloric acid $(\mathrm{HCl})$ was measured by standard four-point probe techniques using a custom made probe setup.

\section{Protein-membrane interaction Atomic force microscopy}

Protein-binding force was quantified by atomic force microscopy (AFM). Fn conjugated AFM probes were prepared and characterized as previously described. ${ }^{[15]}$ Briefly, the probes, (PNP-DB, Nanoworld, Switzerland) with a nominal spring constant of $0.06 \mathrm{~N} / \mathrm{m}$, were $\mathrm{O}_{2}$ plasma treated for $3 \mathrm{~min}$, and then placed into $1 \mathrm{vol} \%$ (ethoxydimethylsilyl)propylamine in toluene for $1 \mathrm{~h}$ at room temperature. The probes were rinsed with toluene, followed by PBS, and immersed in $2.5 \mathrm{vol} \%$ glutaraldehyde in PBS for $1 \mathrm{~h}$ at room temperature. The probes were then rinsed in PBS and immersed in a $10 \mathrm{mg} / \mathrm{mL}$ Fn solution from human plasma for $1 \mathrm{~h}$. The probes were finally rinsed and refrigerated in PBS solution until use. AFM force spectroscopy of Fn adhesion was performed using a Keysight Technologies 5500 AFM (USA). The force spectroscopy measurements were performed across a $40 \times 40 \mu \mathrm{m}^{2}$ area with 64 individual force curves, on three separate locations across the sample. The measurements were repeated with three Fn functionalized probes. Force spectroscopy results were analyzed using PUNIAS software (punias.free.fr). Specifically, the protein unfolding length is measured as the length between two consecutive peaks within the AFM force curve, where the peaks represent a detachment event between the protein and the surface; the total protein unfolding length is calculated as the sum of all the unfolding lengths within each force curve minus the unfolding length between the first two peaks. 


\section{Protein adsorption on the membranes}

Protein adsorption was further quantified through the use of fluorescein conjugated fibronectin (FITC-Fn). Scaffolds of 6 $\mathrm{mm}$ in diameter were placed in black flat bottom 96-well plates, and incubated with $100 \mu \mathrm{L}$ FITC-Fn solution $(10 \mu \mathrm{g} / \mathrm{mL}$ in PBS) for $20 \mathrm{~h}$. Samples were washed three times in PBS and fluorescence intensity measured at $\lambda_{\mathrm{ex}}=475 \mathrm{~nm}$ and $\lambda_{\mathrm{em}}=$ 512 nm (Perkin Elmer EnSpire Plate Reader, USA).

\section{In vitro evaluation}

Electrospun membranes were prepared and fixed in cell culture plates as previously reported. ${ }^{[27]} \mathrm{In}$ brief, scaffolds of $6 \mathrm{~mm}$ diameter were cut out with biopsy punches, and fixed with insect pins on cured poly(dimethyl siloxane) (PDMS). Membranes were sterilized under UV overnight. Mouse pre-osteogenic precursor cells (MC3T3-E1, ATCC, UK) were expanded in minimum essential medium Eagle - alpha modification ( $\alpha$-MEM) supplemented with $10 \mathrm{vol} \%$ FBS and $1 \mathrm{vol} \%$ penicillin/streptomycin. MC3T3-E1 were seeded at a density of 110,000 cells per scaffold and cultured for 3 days in basal media. Osteogenic differentiation was initiated by addition of $\alpha$-MEM supplemented with 10 vol\% HyClone FBS (Thermo Scientific, UK), 1 vol\% penicillin/streptomycin, $5 \mu \mathrm{g} / \mathrm{mL} \mathrm{L}$-ascorbic acid and $2 \mathrm{mM}$ $\beta$-glycerophosphate disodium salt. Media was changed every 2 days over a differentiation period of 21 days. In vitro evaluation was accomplished with $n=3$ individual experiments and three individual scaffolds per experiment per condition.

Cell proliferation was assessed based on DNA quantification. Briefly, constructs were washed in $1 \times$ PBS, cells lysed in distilled water, and three repeated freeze-thawing cycles. DNA was quantified with Hoechst DNA-binding agent. Fluorescence was measured at $\lambda_{\mathrm{ex}}=360 \mathrm{~nm}$ and $\lambda_{\mathrm{em}}=460$ nm (Perkin Elmer EnSpire Plate Reader, USA). Osteogenic differentiation was assessed after 21 days in culture. For qPCR, scaffolds were lysed in RTL buffer, supplemented with 1 vol $\% \beta$-mercaptoethanol. RNA was isolated with an RNA isolation kit (RNeasy Mini Kit, Qiagen, Germany) and cDNA synthesis was accomplished with a reverse transcriptase kit (Reverse Transcriptase Kit, Qiagen, Hilden, Germany). Samples were analyzed for the expression of ALPL, COL1A1, and RUNX2, normalized to the housekeeping gene 18S (all primers from Life Technologies, Thermo Fisher, UK). Sequences of forward and reverse primer are found in Supplementary Table S1. qPCR was run on a QuantStudio6 real-time PCR System (Applied Biosystems, USA). Samples were run with Express SYBR green super mix. Fold expression was calculated as $2^{-\Delta \Delta C t}$ normalized to $18 \mathrm{~S}$ and gene expression on day 0 . Immunohistochemistry was accomplished on formaldehyde-fixed samples. Samples were incubated with anti-osteocalcin antibody (rabbit anti mouse, aa 59-74, MERCK, Millipore, Germany) followed by a fluorescently labeled secondary antibody (anti-rabbit IgG, Alexa 555, Life Technologies, Thermo Fisher, UK). Samples were mounted with glycergel mounting media (Dako, Agilent technologies,
Ely, UK). Images were acquired on a confocal microscope (LSM780, Carl Zeiss AG, Switzerland).

\section{Statistical analysis}

Significant differences were assessed by one way analysis of variance, followed by pairwise comparison with Bonferroni correction. Results were accepted as significantly different for $P<0.05$.

\section{Results \\ Synthesis and electrical characterization of AT-PCL and AT-PCL/PCL membranes}

Following a previously published synthetic route (Supplementary Fig. S1A), AD-PCL with a molecular weight of $\sim 1000 \mathrm{~g} / \mathrm{mol}$ (7 caprolactone units) was synthesized. ${ }^{[22]}$ Subsequently, a second $\mathrm{AD}$ unit was oxidatively coupled to AD-PCL, yielding AT-PCL with a molecular weight of $\sim 1300 \mathrm{~g} / \mathrm{mol}$. Due to the acidic reaction conditions, AT-PCL was obtained in its doped, emeraldine salt (ES) state and was de-doped in $0.1 \mathrm{M} \mathrm{NH}_{4} \mathrm{OH}$, yielding a dark blue/purple product in its emeraldine base (EB) state (Supplementary Fig. S1).

To investigate electrical conductivity, four-point probe measurements were accomplished on HCl-doped electrospun membranes (detailed below). At increasing ratios of AT-PCL to PCL from $25 \%$ to $75 \%$, a reduced sheet resistance was observed $\left(24.7 \pm 5.6 \times 10^{6}, 7.1 \pm 1.7 \times 10^{6}\right.$, and $3.0 \pm 0.6 \times 10^{6} \Omega / \mathrm{sq}$, respectively), indicating increased electrical conductivity [Fig. 1(a)]. However, the instability of $\mathrm{HCl}$ doping led to immediate de-doping when immersed in physiological buffer (PBS). This prevents its use as dopant for cell culture membranes, where stable doping is required. Phytic acid has been reported as a stable dopant for poly(aniline), ${ }^{[28]}$ and we therefore hypothesized that this non-toxic acid would work comparably in the herewith designed system. The optoelectronic activity of AT-PCL doped with phytic acid was confirmed by UV-VIS absorbance spectrometry (Fig. 1). The insulating EB state has a characteristic absorption maximum at $\sim 600 \mathrm{~nm}$, attributed to the excitonic transition $\pi_{\mathrm{B}}-\pi_{\mathrm{Q}}$ of benzenoid (B) to quinoid (Q). Upon the addition of phytic acid AT-PCL was doped to the ES state, resulting in the emergence of an additional adsorption maximum at $\sim 400 \mathrm{~nm}$, characteristic of bipolarons. Interestingly, however, these changes in oxidation and doping were not reflected in four-point probe electrical measurements. Electrospun membranes, as described below, of AT-PCL blended with PCL and doped with $0.02 \mathrm{M}$ phytic acid did not conduct any measurable current, and sheet resistance could not be determined at the applied voltage, attributed to the weak acidic properties of phytic acid and its minor doping capabilities compared with stronger acids such as $\mathrm{HCl}^{[28]}$

Aniline tetramer has previously been shown to exhibit similar redox properties to PANi. ${ }^{[19]}$ As a co-polymer with PCL these properties were maintained and AT-PCL was shown to retain the characteristic properties of the aniline oligomer. At phytic acid concentrations as low as $0.005 \mathrm{M}$, we could demonstrate the optoelectronic activity of AT-PCL. This warranted further 


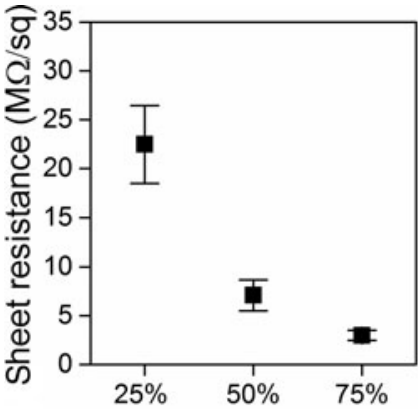

(a)

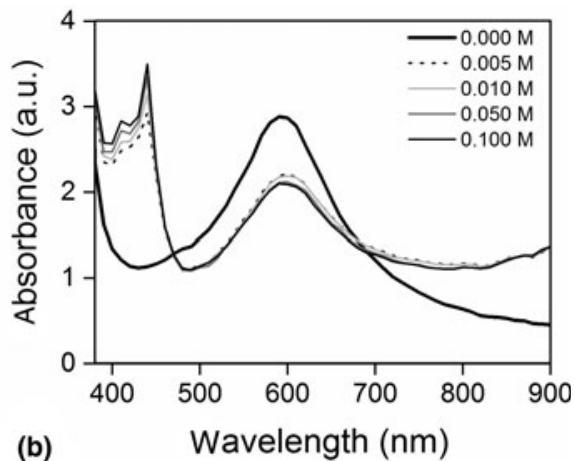

FIG. 1. Electrical and optoelectronic characterization of AT-PCL. (a) Sheet resistance of electrospun membranes, produced at different AT-PCL to PCL ratios and doped with $0.1 \mathrm{M} \mathrm{HCl}$. (b) Spectra of AT-PCL in DMSO doped with phytic acid at different molarities.

in vitro evaluation and the study of protein adsorption on membranes as a function of AT-PCL/PCL ratios and doping state.

\section{Electrospinning}

Fibrous scaffolds prepared by electrospinning have proven particularly versatile for the design of biografts due to their close architectural resemblance to the fibrous ECM, and they have therefore been widely applied in tissue engineering. However, this method cannot typically be applied to fully conjugated systems, due to their poor solubility in organic solvents. Conjugated oligomers, and in particular end functionalized oligomers or block-co-polymers offer opportunities to address this problem, whereby the solubility of an insulating partner can be utilized to modify the system properties. However, electrospinning of aniline tetramer or pentamer based materials for bone tissue engineering has been restricted to very few publications, e.g. Liu et al. ${ }^{[7]}$ in which the conductive unit was grafted to a gelatin-PLLA polymer. As an alternative to chemical grafting, blending polymer systems have emerged as a versatile method to combine distinct material properties during the process of electrospinning. To this end, AT-PCL was blended with high molecular weight PCL (70,000-90,000 Da) and doped by adding phytic acid to the polymer solution prior to electrospinning (final concentration of $0.02 \mathrm{M}$ in $\mathrm{CHCl}_{3}: \mathrm{CH}_{3} \mathrm{OH}$ ). Doped solutions turned green immediately, indicative of the ES state of AT-PCL, whereas non-doped solutions retained the dark purple color characteristic of the EB state. Electrospinning of AT-PCL/PCL blends in a $9: 1$ mixture of $\mathrm{CHCl}_{3}: \mathrm{CH}_{3} \mathrm{OH}$ resulted in the deposition of fibrous, nonwoven, free standing mats, of blue or green color depending on the doping state. Fiber diameters were measured on scanning electron microscopy (SEM) images and the mean fiber diameter was calculated based on 100 individual measurements. For all blending ratios, these were in the sub-micrometer range. For pristine membranes, mean fiber diameters were $0.4 \pm 0.1 \mu \mathrm{m}$ (25\% AT-PCL); $0.3 \pm 0.1 \mu \mathrm{m}$ (50\% AT-PCL); $0.3 \pm 0.1 \mu \mathrm{m}(75 \%$ AT-PCL), while doped membranes displayed values of $0.3 \pm 0.1 \mu \mathrm{m}(25 \%$ AT-PCL $) ; 0.4 \pm 0.1 \mu \mathrm{m}$
(50\% AT-PCL); $0.3 \pm 0.1 \mu \mathrm{m} \quad(75 \%$ AT-PCL) (Fig. 2). Electrospun membranes retained their color when immersed in PBS or cell culture media for several hours, indicating stable doping, which significantly surpassed that of $\mathrm{HCl}$-doped membranes. AT-PCL/PCL ratios of $50 \%$ or $75 \%$ resulted in the occasional formation of beaded membranes, due to lower solution viscosity. In the doped state, however, increased electrical conductivity resulted in the deposition of homogenous fibers.

After in vivo implantation or in vitro cell culture, a protein layer is adsorbed on the material within the first minutes or hours, then regulates further downstream signals for cell adhesion, cell proliferation, and/or differentiation. ${ }^{[11]}$ We propose that this stable doping for several hours is sufficient to significantly alter protein adsorption and concomitant cell response, and therefore represents an important strategy for the design of electrically active $3 \mathrm{D}$ scaffolds for tissue engineering.

\section{Protein-membrane interaction}

Controlling protein adsorption via material properties is a powerful means to tune bio-interfaces and steer material-cell interactions. We were therefore interested in protein adsorption on doped versus pristine substrates, following previously published reports that the oxidative state of a conjugated polymer may induce significant changes in both protein adsorption levels and conformation. ${ }^{[12,13,15]}$ As a major constituent of native ECM, we investigated Fn-membrane interactions with atomic force spectroscopy. The required force and work to pull off the protein from the membrane, and the protein's length at release are displayed in Fig. 3. On doped samples of $50 \%$ or $75 \%$ AT-PCL, significantly higher forces were required to pull Fn when compared with pristine membranes at the same concentration. Similarly, significantly longer Fn length was observed on pristine 50\% AT-PCL compared with doped $50 \%$ AT-PCL. Protein length was also shown to be significantly longer on membranes containing 25\% AT-PCL compared with $50 \%$ or $75 \%$. These results indicate reduced protein-membrane surface interactions on doped substrates, implying a change in protein conformation on the surfaces of 
(a)

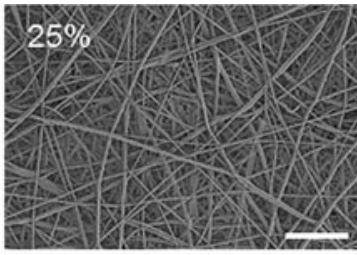

(b)

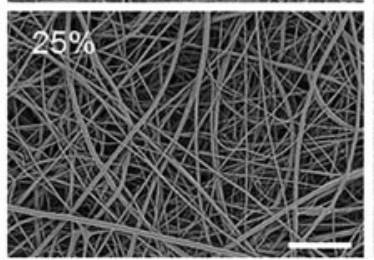

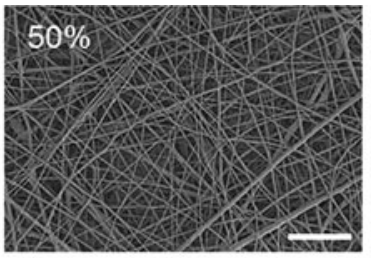
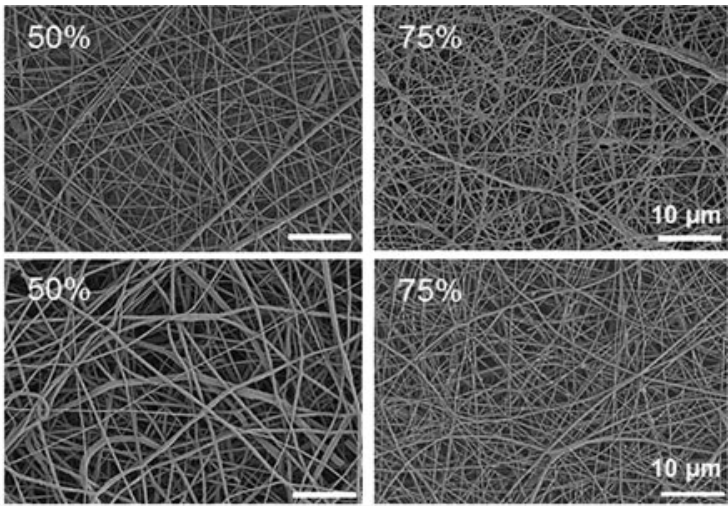

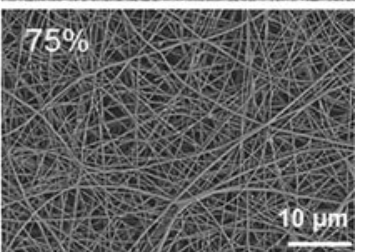

FIG. 2. SEM images of fibrous membranes. AT-PCL was blended at different w/w ratios (25\%, $50 \%$, and $75 \%)$ with high molecular weight PCL and processed by electrospinning. For all AT-PCL to PCL ratios, free standing membranes were produced. (a) Pristine AT-PCL membranes, (b) phytic acid-doped AT-PCL membranes.
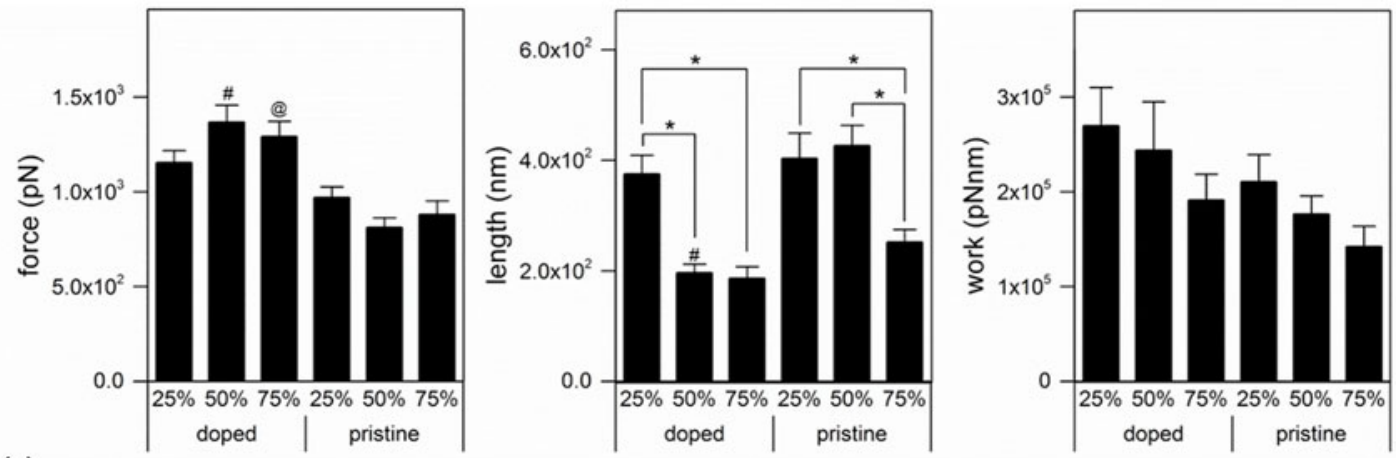

(a)

(i)

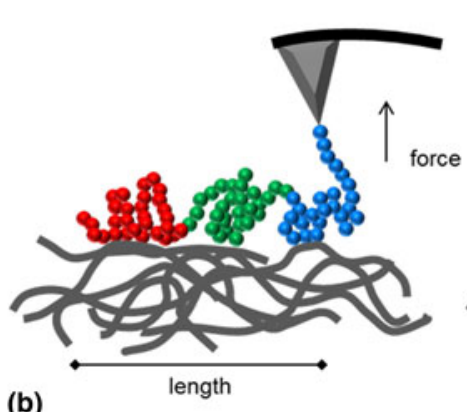

(b)

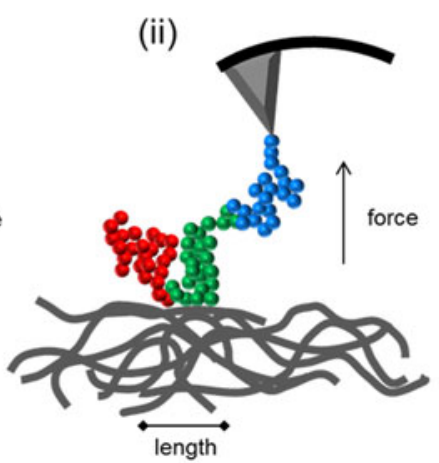

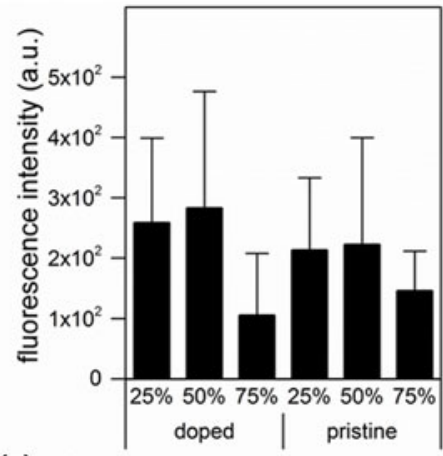

(c)

FIG. 3. (a) Fibronectin-membrane interactions recorded with atomic force microscopy (AFM). Force recorded to remove Fn from the substrate, calculated length of the folded/unfolded protein, and work required to remove Fn from the substrate. Results are presented as mean $\pm \mathrm{SE}$ with ${ }^{*} P<0.05$; ${ }^{\#} P<0.05$ compared with 50\% AT-PCL; ${ }^{\circledR} P<0.05$ compared with 75\% AT-PCL. (b) Schematic representation of the results obtained in (a). (i) Pristine samples: an increased length is indicative for an extended Fn conformation, with larger contact area with the substrate, whereas in (ii) (doped samples), a shorter length indicates a more folded, compact Fn conformation, with reduced contact area. (c) Quantification of FITC-Fn adsorption on the respective substrates. Results are presented as mean \pm SD. 
doped substrates compared with pristine ones. The observed protein lengths are longer than a single Fn protein $(160 \mathrm{~nm})$, which can be attributed to the 3D nature of the electrospun polymer fibers. As the entire AFM probe has been functionalised with Fn, multiple proteins on the apex and side of the probe may bind to the fibers, contributing to longer unbinding lengths.

We therefore hypothesize that the electrochemical properties of the substrate have a significant effect on protein conformation, with Fn being in a more relaxed, extended conformation on pristine samples, exposing a larger portion of protein binding sites to the membrane, resulting in longer binding length. ${ }^{[12,13]}$ Reduction of the conjugated system to the ES state results in different protein adhesion, as a result of reduced protein-membrane contact (length) induced by a more coil-like folding of Fn. The amount of adsorbed Fn was, however, similar on all substrates, as indicated by the fluorescence intensity of bound FITC-Fn (Fig. 3). We therefore conclude that while the oxidative state of conjugated membranes does not control absolute protein adsorption concentration, it does have a significant effect on conformation. This change in protein conformation could have a significant effect on cell adhesion and further downstream events. $^{[14]}$

\section{In vitro evaluation}

As a model system for osteogenesis in vitro, we assessed osteogenic differentiation of MC3T3-E1 osteogenic precursor cells on the different membranes. Despite phytic acid doping not resulting in membranes of measurable conductivity, we hypothesized the influence of doping on protein conformation would induce a distinct response on MC3T3-E1 differentiation. Intercellular signaling or charge transport might furthermore be influenced by the material properties, warranting the evaluation of our membranes in vitro. DNA content, based on fluorescent spectroscopy after Hoechst staining, remained constant over a period of 21 days and no significant differences in DNA content were observed between different substrates (Supplementary Fig. S3). Our results therefore demonstrate that AT-PCL membranes are suitable cell culture substrates for long-term studies of up to 21 days under differentiation conditions, without any major decrease in cell number. This suggests that even at higher relative concentrations of $75 \%$ AT-PCL, the conjugated oligomer does not have a cytotoxic effect. Furthermore, these long-term studies show that phytic acid can be used as a non-toxic dopant.

Gene expression analysis of key osteogenic markers was undertaken by qPCR (Fig. 4). ALPL, COL1A1, and RUNX2 expression was increased on day 21 compared with day 3 on all substrates. Of note, on doped 50\% AT-PCL and on pristine 25\% AT-PCL, significantly higher COL1A1 expression was found on day 21 compared with day 3. Additionally, RUNX2 expression on pristine $25 \%$ AT-PCL was significantly higher than any other condition, in particular when compared with doped $25 \%$ AT-PCL or pristine $75 \%$ AT-PCL. On all other samples, differences between days 21 and 3 were not statistically significant. Osteocalcin deposition, as assessed by immunohistochemistry and confocal microscopy (Fig. 5), supported the trend of increased osteogenic differentiation on $25 \%$ ATPCL. Increased positive staining for osteocalcin was observed on pristine membranes, with enhanced positive staining on $25 \%$ AT-PCL compared with $50 \%$ or $75 \%$ AT-PCL.

The in vitro evaluation suggests that $25 \%$ AT-PCL membranes are most promising candidates for differentiation of MC3T3-E1 osteogenic precursor cells. The underlying mechanisms are still elusive, yet an altered protein conformation, as indicated by an increased Fn length upon removal (which is indicative for a more elongated, harmonica-like protein conformation), could have affected initial cell attachment, further ECM secretion of MC3T3-E1 and concomitant differentiation. Overall, these results indicate that osteogenic differentiation on membranes of higher AT-PCL content is not fully supported, and no osteo-inductive properties can be attributed to AT-PCL membranes. Limited reports on in vitro evaluation of scaffolds based on conjugated aniline oligomers lie in strong contrast with the vast amount of publications on synthesis routes, and our results can only marginally be related to previous work. While Liu et al. reported on the design of electrospun scaffolds containing aniline pentamer, with enhanced
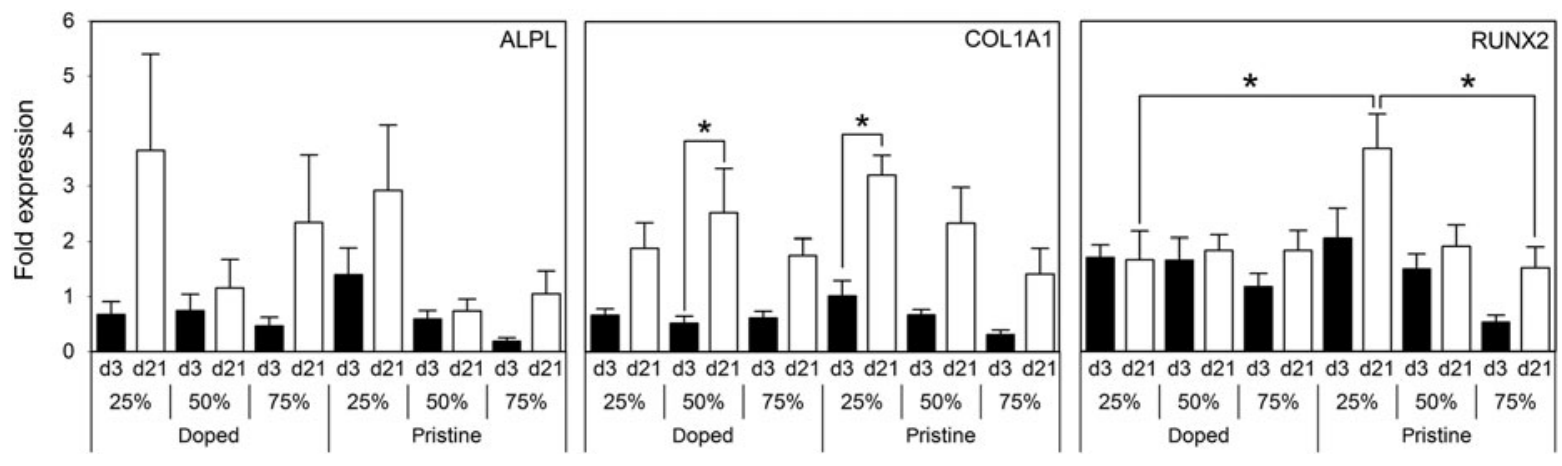

FIG. 4. Gene expression of osteogenic genes ALPL, COL1A1, and RUNX2. Results are presented as mean $\pm \mathrm{SE}$ with ${ }^{*} P<0.05$. 

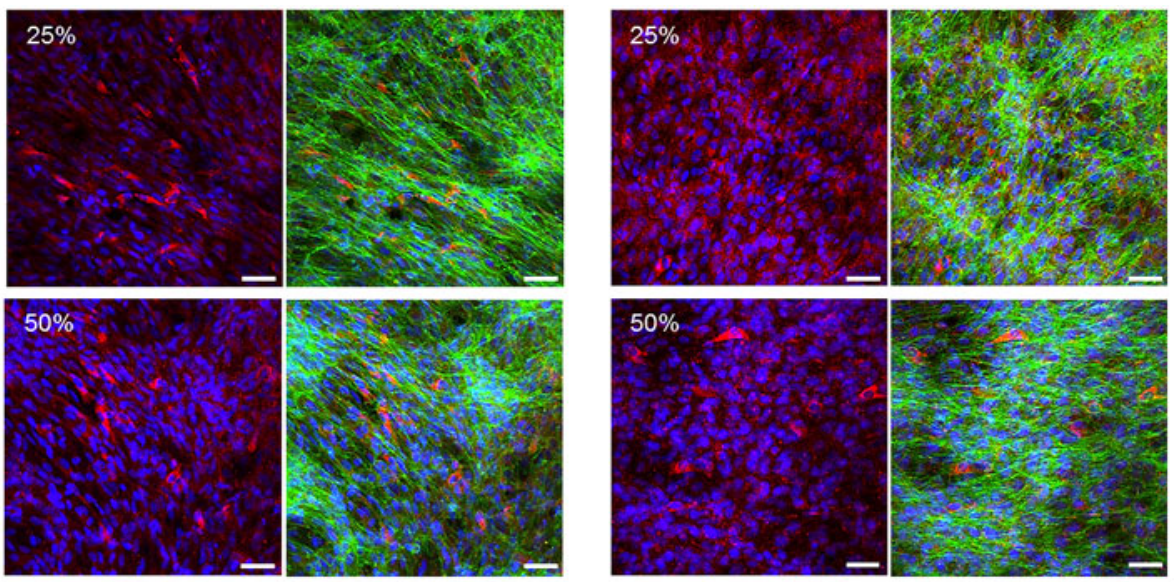

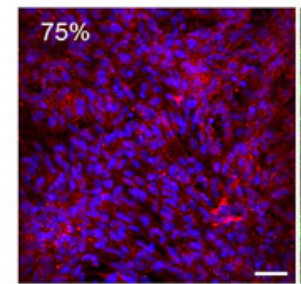

(a)

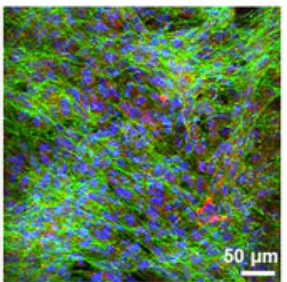

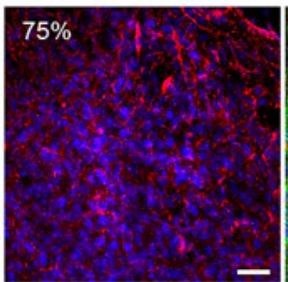

(b)

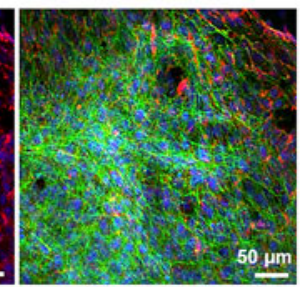

FIG. 5. Confocal microscopy images of MC3T3-E1 cultured on electrospun membranes of AT-PCL for 21 days under osteogenic differentiation conditions. Cells were stained for osteocalcin (red), actin (green), and nuclei (blue). (a) Phytic acid-doped membranes, (b) pristine membranes.

MC3T3-E1 proliferation compared with PLLA scaffolds alone, no qPCR was performed and it remains open to which extent conductive scaffolds induced enhanced gene expression of osteogenic markers. ${ }^{[7]}$

In summary, our AT-PCL scaffolds did allow for cell attachment and supported culture of MC3T3-E1 over 21 days, allowing us to demonstrate cytocompatibility and longterm performance of AT-PCL scaffolds in vitro. The cytotoxicity and biodegradation of aniline oligomers is still the subject of controversial discussion and the importance of functional endgroups has been emphasized to be influential in this regard. ${ }^{[17,29,30]}$ Our study further underpins the necessity for sophisticated and highly reliable in vitro models for assessing the cytocompatibility and biodegradability of aniline oligomerbased scaffolds. The high variability observed among experimental repeats during this and prior research highlights the need for accurately controlled synthetic routes to conjugated materials, as a vital step towards the design of scaffolds that allow experimental reproducibility and consistency. Furthermore, analytical methods to assess optoelectronic properties and electrical conductivities need to be optimized to be applied to 3D porous culture substrates. In this respect, oligomeric materials, in contrast to polymers, are particularly attractive due to their potential to be synthesized under highly controlled conditions, resulting in standardized and reproducible chain length and chemical properties. ${ }^{[18]}$ As such, we envisage that such areas of research will find increasing interest in the near future.

\section{Conclusions}

In summary, we created electropsun scaffolds of distinct AT-PCL content and showed their cytocompatibility in MC3T3-E1 pre-osteogenic cell culture. In a blend system, we demonstrated that AT-PCL is spinnable, allowing for the production of electrically active scaffolds of otherwise highly difficult to process conjugated polymers. In this respect, the use of oligomers, and blending with high molecular weight PCL was required to enable electrospinning. Depending on the content and redox state of the conjugated block, a distinct protein adsorption profile was observed, indicating conformational changes of Fn upon adhesion to doped or pristine (nondoped) samples. Our results further highlight the importance of highly controlled chemical synthesis, scaffold design and reliable in vitro models for studying cell-material interactions and design $3 \mathrm{D}$ cell culture substrates.

\section{Supplementary material}

The supplementary material for this article can be found at https://doi.org/10.1557/mrc.2017.45.

\section{Acknowledgements}

We would like to thank Dr. Robert Chapman, Imperial College London, for the synthesis of FITC-conjugated Fibronectin. A. G. G. is grateful to fellowships from the Swiss National Science Foundation (Grant No. P2BEP3_152091 and P300PB_ 161072). A. G. G., C. D. S., E. J. H., C. M. T., S. E. H., 
and M.M.S. acknowledge the British Heart Foundation Cardiovascular Regenerative Medicine Centre (RM/13/1/ 30157). A. A. was supported by a Ph.D. studentship of the EPSRC Centre for Doctoral Training under the Centre for Plastic Electronics, Imperial College London (EP/G037515/ 1). A. G. acknowledges the support from the H2020 through the Individual Marie Skłodowska-Curie Fellowship 'RAISED' under grant agreement no. 660757. M. M. S. was supported by the UK Regenerative Medicine Platform Hubs 'Acellular Approaches for Therapeutic Delivery' (MR/K026682/1). M. M. S. acknowledges the grant 'State of the Art Biomaterials Development and Characterization of the Cell-Biomaterial Interface' (MR/L012677/1) from the MRC. M. M.S. also acknowledges the support from the ERC Seventh Framework Programme Consolidator grant 'Naturale CG' (616417). Research raw data is available online at DOI: 10.5281/ zenodo.806182.

\section{Conflict of interest}

The authors declare no competing financial interest.

\section{Author contributions}

A. G. G. designed the study, conducted experiments, performed data analysis and wrote the manuscript. C. D. S. supported study design, performed data analysis and revised the manuscript. A. G., A. A., and E. J. H. helped with experiments and data analysis. C. M. T. and S. E. H. provided valuable inputs to the study design and revised the manuscript. M. M. S. supervised the study, participated in scientific discussions, and revised the manuscript.

\section{References}

1. A.R. Amini, C.T. Laurencin, and S.P. Nukavarapu: Bone tissue engineering: recent advances and challenges. Crit. Rev. ${ }^{T M}$ Biomed. Eng. 40, 363-408 (2012).

2. S. Bose, M. Roy, and A. Bandyopadhyay: Recent advances in bone tissue engineering scaffolds. Trends Biotechnol. 30, 546-554 (2012).

3. X. Yu, X. Tang, S.V. Gohil, and C.T. Laurencin: Biomaterials for bone regenerative engineering. Adv. Healthc. Mater. 4, 1268-1285 (2015).

4. E.S. Place, N.D. Evans, and M.M. Stevens: Complexity in biomaterials for tissue engineering. Nat. Mater. 8, 457-470 (2009).

5. J.G. Hardy, J.Y. Lee, and C.E. Schmidt: Biomimetic conducting polymerbased tissue scaffolds. Curr. Opin. Biotechnol. 24, 847-854 (2013).

6. H. Cui, Y. Liu, M. Deng, X. Pang, P. Zhang, X. Wang, X. Chen, and Y. Wei: Synthesis of biodegradable and electroactive tetraaniline grafted poly(ester amide) copolymers for bone tissue engineering. Biomacromolecules 13, 2881-2889 (2012)

7. Y. Liu, H. Cui, X. Zhuang, Y. Wei, and X. Chen: Electrospinning of aniline pentamer-graft-gelatin/plla nanofibers for bone tissue engineering. Acta Biomater. 10, 5074-5080 (2014).

8. R. Balint, N.J. Cassidy, and S.H. Cartmell: Conductive polymers: towards a smart biomaterial for tissue engineering. Acta Biomater. 10, 2341-2353 (2014).

9. A.D. Bendrea, L. Cianga, and I. Cianga: Review paper: progress in the field of conducting polymers for tissue engineering applications. J. Biomater. Appl. 26, 3-84 (2011).

10. T.H. Qazi, R. Rai, and A.R. Boccaccini: Tissue engineering of electrically responsive tissues using polyaniline based polymers: a review. Biomaterials 35, 9068-9086 (2014).
11. D.G. Castner and B.D. Ratner: Biomedical surface science: foundations to frontiers. Surf. Sci. 500, 28-60 (2002).

12. M.J. Higgins, P.J. Molino, Z. Yue, and G.G. Wallace: Organic conducting polymer-protein interactions. Chem. Mater. 24, 828-839 (2012).

13.P.J. Molino, M.J. Higgins, P.C. Innis, R.M. Kapsa, and G.G. Wallace: Fibronectin and bovine serum albumin adsorption and conformational dynamics on inherently conducting polymers: a qcm-d study. Langmuir. 28, 8433-8445 (2012).

14. A.M. Wan, R.M. Schur, C.K. Ober, C. Fischbach, D. Gourdon, and G.G. Malliaras: Electrical control of protein conformation. Adv. Mater. 24, 2501-2505 (2012).

15. A. Gelmi, M. Higgins, and G.G. Wallace: Resolving sub-molecular binding and electrical switching mechanisms of single proteins at electroactive conducting polymers. Small 9, 393-401 (2013).

16. B. Guo, L. Glavas, and A.-C. Albertsson: Biodegradable and electrically conducting polymers for biomedical applications. Prog. Polymer Sci. 38, 1263-1286 (2013).

17. T.J. Rivers, T.W. Hudson, and C.E. Schmidt: Synthesis of a novel, biodegradable electrically conducting polymer for biomedical applications. Adv. Funct. Mater. 12, 33-37 (2002)

18.C.D. Spicer, M.A. Booth, D. Mawad, A. Armgarth, C.B. Nielsen, and M.M. Stevens: Synthesis of hetero-bifunctional, end-capped oligo-edot derivatives. Chemistry 2, 125-138 (2017).

19. R.L. Li, C.-W. Lin, Y. Shao, C.W. Chang, F.-K. Yao, M.D. Kowal, H. Wang, M.T. Yeung, S.-C. Huang, and R.B. Kaner: Characterization of aniline tetramer by maldi tof mass spectrometry upon oxidative and reductive cycling. Polymers 8, 401 (2016).

20. H. Cui, J. Shao, Y. Wang, P. Zhang, X. Chen, and Y. Wei: Pla-peg-pla and its electroactive tetraaniline copolymer as multi-interactive injectable hydrogels for tissue engineering. Biomacromolecules 14, 1904-1912 (2013).

21. H. Cui, Y. Liu, Y. Cheng, Z. Zhang, P. Zhang, X. Chen, and Y. Wei: In vitro study of electroactive tetraaniline-containing thermosensitive hydrogels for cardiac tissue engineering. Biomacromolecules 15, 1115-1123 (2014).

22. B. Guo, A. Finne-Wistrand, and A.-C. Albertsson: Universal two-step approach to degradable and electroactive block copolymers and networks from combined ring-opening polymerization and post-functionalization via oxidative coupling reactions. Macromolecules 44, 5227-5236 (2011)

23. H. Qi, M. Liu, L. Xu, L. Feng, L. Tao, Y. Ji, X. Zhang, and Y. Wei: Biocompatibility evaluation of aniline oligomers with different endfunctional groups. Toxicol. Res. 2, 427-433 (2013).

24. Y. Liu, H. Cui, X. Zhuang, P. Zhang, Y. Cui, X. Wang, Y. Wei, and X. Chen: Nano-hydroxyapatite surfaces grafted with electroactive aniline tetramers for bone-tissue engineering. Macromol. Biosci. 13, 356-365 (2013).

25. Q. Wang, Q. Wang, and W. Teng: Injectable, degradable, electroactive nanocomposite hydrogels containing conductive polymer nanoparticles for biomedical applications. Int. J. Nanomed. 11, 131 (2016)

26. P. Campagnolo, A.J. Gormley, L.W. Chow, A.G. Guex, P.A. Parmar, J.L. Puetzer, J.A. Steele, A. Breant, P. Madeddu, and M.M. Stevens: Pericyte seeded dual peptide scaffold with improved endothelialization for vascular graft tissue engineering. Adv. Healthc. Mater. 5, 30463055 (2016).

27. A.G. Guex, G. Fortunato, D. Hegemann, H.T. Tevaearai, and M.N. Giraud: General protocol for the culture of cells on plasma-coated electrospun scaffolds. Methods Mol. Biol. 1058, 119-131 (2013).

28. D. Mawad, C. Mansfield, A. Lauto, F. Perbellini, G.W Nelson, S.0 Bello, D.J. Carrad, A.P. Micolich, M.M. Mahat, J. Furman, A.R. Lyon, J.J. Gooding, S.E. Harding, C.M. Terracciano, and M.M. Stevens: A conducting polymer with enhanced electronic stability applied in cardiac models. Sci. Adv. 2, e1601007 (2016).

29. X. Zhang, H. Qi, S. Wang, L. Feng, Y. Ji, L. Tao, S. Li, and Y. Wei: Cellular responses of aniline oligomers: a preliminary study. Toxicol. Res. 1, 201$205(2012)$

30. L. Song, B. Du, L. Chen, M. Deng, H. Sun, X. Pang, P. Zhang, and $X$. Chen: Synthesis of electroactive and biodegradable multiblock copolymers based on poly(ester amide) and aniline pentamer. J. Polymer Sci. A: Polymer Chem. 51, 4722-4731 (2013). 